\title{
Evaluation of the spatial arrangement of Purkinje cells in ataxic rat's cerebellum after Sertoli cells transplantation
}

\author{
R. Mohammadi ${ }^{1}$, M.H. Heidari ${ }^{1}$, Y. Sadeghi' ${ }^{1}{ }^{2}$, M.-A. Abdollahifar ${ }^{1}$, A. Aghaei ${ }^{1}$ \\ ${ }^{1}$ Department of Anatomy, Faculty of Medicine, Shahid Beheshti University of Medical Sciences, Tehran, Iran \\ ${ }^{2}$ Hearing Disorder Research Centre, Shahid Beheshti University of Medical Sciences, Tehran, Iran
}

[Received: 24 June 2017; Accepted: 29 September 2017]

Background: Purkinje cells (PCs) pathology is important in cerebellar disorders like ataxia. The spatial arrangement of PCs after different treatments has not been studied extensively. Immunohistochemistry (IHC) analysis of cerebellum can give a proper tool for explaining the pathophysiology of PCs in ataxia. Here we stereologically analysed the 3-dimensional spatial arrangement of PCs in the cerebellum of rats after ataxia induction with 3-acetylpyridine (3-AP).

Materials and methods: Ataxia was induced in rats by intraperitoneal injection of 3-AP $(65 \mathrm{mg} / \mathrm{kg})$. Spatial arrangement of PCs for differences in ataxic rats with (3-AP-SC) or without (3-AP) Sertoli cells (SCS) transplantation was evaluated using second-order stereology. The IHC method by using antibodies to anti-calbindin in the cerebellum was applied.

Results: Our results showed that a random arrangement is at larger distances between PCs in 3-AP and 3-Ap-SC groups. Therefore the PCs are not normally arranged after 3-AP and SCs transplantation stored the spatial arrangements of the cells after ataxia induction in rats. $I H C$ analyse shows that number of PCS was significantly improved after the SC transplantation.

Conclusions: Segregation of PCs can be observed at some areas in the ataxic rats' cerebellum. However, the spatial arrangement of PCs was unchanged in SCS transplanted rats. (Folia Morphol 2018; 77, 2: 194-200)

Key words: ataxia, 3-acetylpyridine, cell transplantation, Sertoli cells, spatial arrangement analysis, Purkinje cells

\section{INTRODUCTION}

The cerebellum is a centre of coordination in the body that receives information from other components of the central nervous system (CNS) [15]. Cerebellar ataxias are a group of neurodegenerative disorders that are identified by progressive damage to the cerebellum [14]. Systemic injection of 3-acetylpyridine (3-AP) induces cerebellar ataxia which characterised by selective degenerative changes in inferior olivary nucleus (ION) neurons and climbing fibres (CF) of olivocerebellar track that innervate the cerebellar Purkinje neurons. Ataxia has significant effects on the cellular integrity, activity and morphology of neurons in the cerebellum $[1,6,9]$.

Cell therapy is a promising treatment to replace degenerated cells and has some trophic effects [4]. Transplantation of some stem cells has been tested in the treatment of ataxia $[5,16]$. Immunosuppression

Address for correspondence: Dr. Y. Sadeghi, Department of Biology and Anatomy School of Medicine Shahid Beheshti Medical University, P.O. Box 19395-4719 Tehran, Iran, tel: +98 21 23872555, fax: + 98212235 3310, e-mail: yousefsadeghi@yahoo.com; yousef.sadeghi@monash.edu 
is required in cell transplantation to prevent rejection of transplanted cells. Because of side effects of immunosuppressants, a reasonable strategy to inhibit cell rejection is utilisation of immune-privileged sites as a source of cells. Immune-privileged sites in the body are the parts in which immune system is weakened and transplanted cells can survive for a long time without arousing the immune system [3].

Sertoli cells (SCs) in testis are immune-privileged cells that provide immune support for spermatogenesis [13]. Some studies suggest that SCs secrete trophic factors following transplantation which can promote cell survival after transplantation [20]. SCs transplantation is a promising approach for treating neurodegenerative diseases like Parkinson [12] and Huntington disease [21].

Stereological methods can provide additional information about the spatial arrangement and structural changes in the cells or organelles in different normal and pathologic conditions. This method has been used to analyse the spatial pattern in different tissues by evaluation of mathematical function $[17,18]$.

Except few reports on the quantitative aspects of the Purkinje cells (PCs), limited studies have been performed on the spatial arrangement of the cerebellum in ataxia. Based on documented survival of SCS transplantation in the different disease, we prompted to examine the effects of SCs transplantation in ataxia on the spatial arrangement of PCs in the cerebellum of rats.

\section{MATERIALS AND METHODS}

\section{Animal models of cerebellar ataxia}

Rats (Wistar/Sprague-Dawley) of 220-240 g weight were used for ataxia model. Experimental procedures and housing were performed according to the policy guidelines of the National Guide for Institutes of Health for the Care and Use of Laboratory (NIH Publications No. 80-23). Rats were randomly divided into control, sham, 3-AP without and 3-AP with SCs transplantation (3-AP-SC) groups. To induce cerebellar ataxia model, rats were given an intraperitoneal injection (IP) of 3-AP (65 mg/kg) in 3-AP and 3-AP-SC groups [9]. This provokes damage to the ION neurons and subsequently PCs in the cerebellum lose their inputs from $\mathrm{CF}$, and the rats suffer from ataxia.

\section{Isolation and culture of testicular cells}

Sertoli cells were isolated from testis of 16-day-old male rats. Following decapsulation, enzymatic digestion was performed using $0.25 \%$ trypsin (Sigma) and
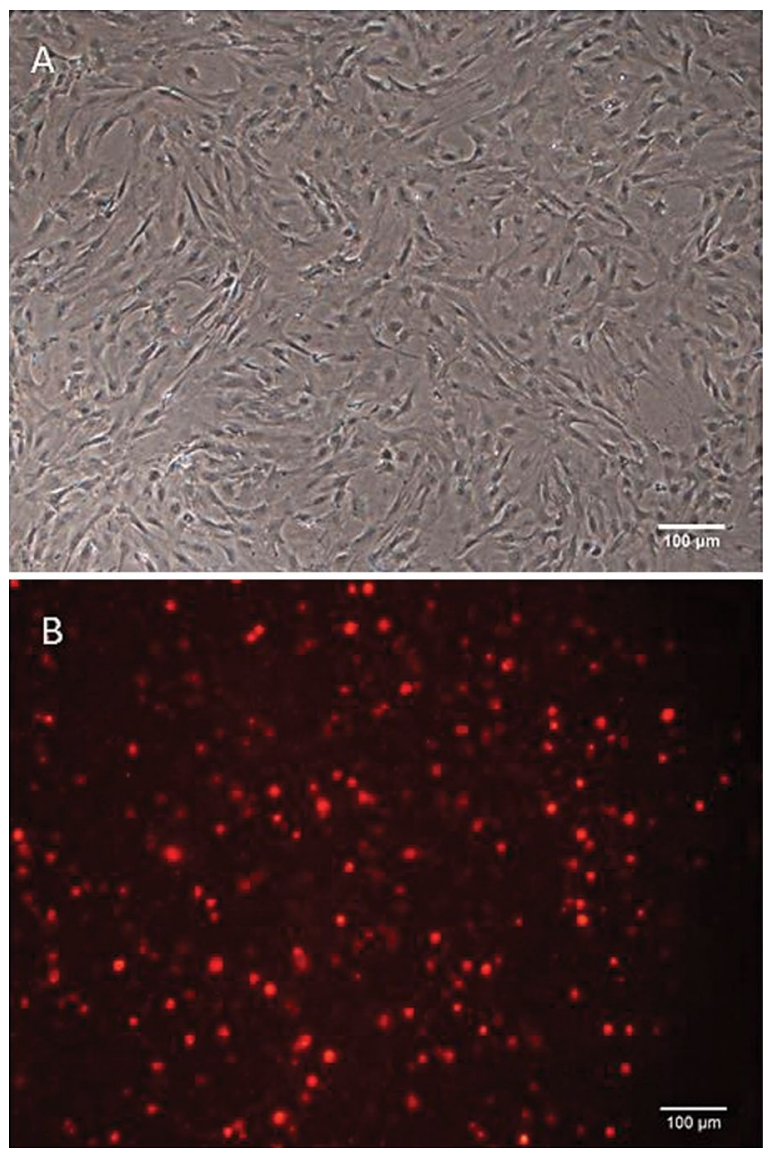

Figure 1. Sertoli cells culture (A). Dil (1,1'-dioctadecyl-3,3,3',3'-tetramethyl-indocarbocyanine perchlorate) labelling of Sertoli cells (B).

$0.1 \%$ collagenase (Sigma, type V) at $37^{\circ} \mathrm{C}$. Isolated cells were transferred into cell culture flasks and kept in the cell culture incubator. After $24 \mathrm{~h}$ the flasks were washed to eliminate debris and medium (DMEM/F12) were changed. After $48 \mathrm{~h}$ the cells were treated for $2.5 \mathrm{~min}$ with hypotonic buffer $(20 \mathrm{mM}$ Tris- $\mathrm{HCl} ; \mathrm{pH}$ 7.4) to lyse any residual germ cells and to get SCs with the purity of $>95 \%$.

\section{Labelling of SCs with Dil}

We applied Dil (1, '1'-dioctadecyl-3,3,3',3'-tetramethyl-indocarbocyanine perchlorate) solutions for labelling of SCs. First, $5 \mu \mathrm{L}$ of Dil solution was added to $1 \mathrm{~mL}$ of serum-free cell suspension $\left(1 \times 10^{6} / \mathrm{mL}\right)$. Then it was incubated for $20 \mathrm{~min}$ at $37^{\circ} \mathrm{C}$ to prepare uniform labelling. Following the centrifugation and re-suspension of cells in a warm medium, labelled SCs suspension was ready for transplantation (Fig. 1). 


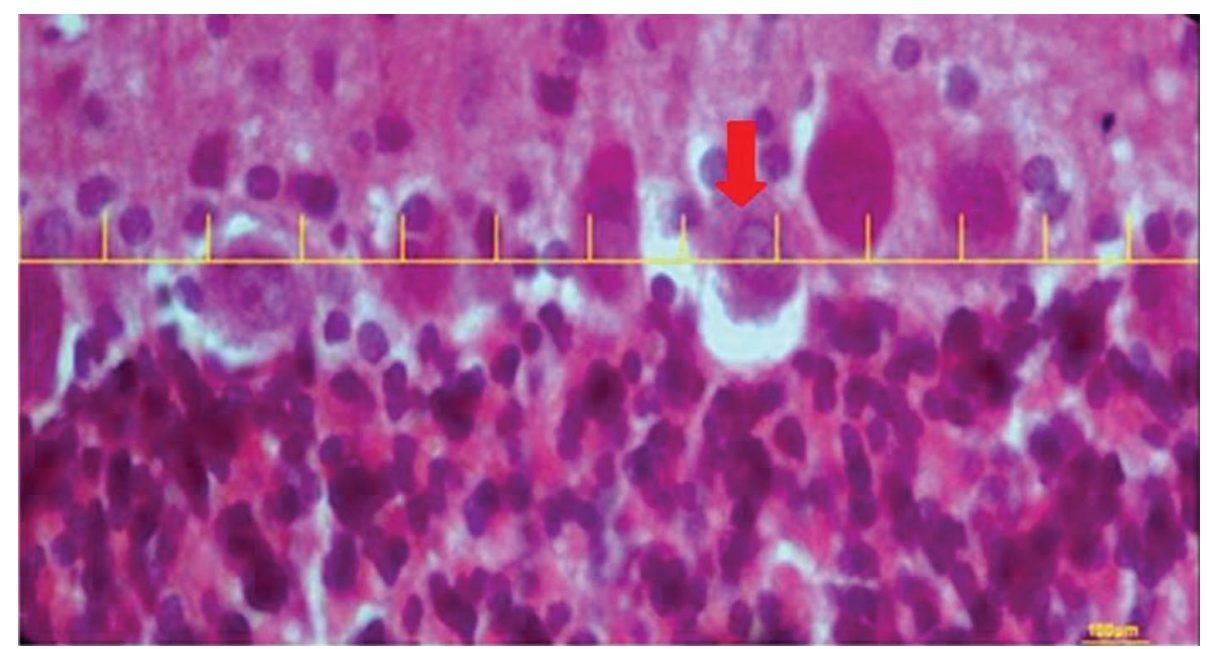

Figure 2. Estimation of spatial arrangement. A transparent lattice bearing one row of points was constructed to serve as a set of dipole probes. The point interval (r) corresponded to a distance of $2.6 \mu \mathrm{m}$. Each test point was coded as 1 and 2 if the point was laid on the Purkinje cells (arrow) and neuropil, respectively.

\section{Transplantation}

Rats in a 3-AP-SC group were anesthetised with an IP administration of ketamine $(100 \mathrm{mg} / \mathrm{kg})$ and xylazine $(5 \mathrm{mg} / \mathrm{kg})$. Then animals were fixed on the stereotaxic frame. The transplantation site was located $12.00 \mathrm{~mm}$ posterior to bregma and $3 \mathrm{~mm}$ lateral to the sagittal suture at a depth of $4 \mathrm{~mm}$ under the skull surface [19]. Infusion of $5 \mu \mathrm{L}$ of SCs suspension $\left(1 \times 10^{4}\right.$ cells $\left./ \mu \mathrm{L}\right)$ was injected over 3-5 min per cerebellar hemisphere. To evaluate the effect of operation on rats, a sham group was treated with injection of the same volume medium (DMEM/F12) without SCs, with same operation and injection condition as 3-AP-SC group.

\section{Tissue preparation and staining}

Six weeks after the 3-AP injection, the rats were anesthetised with ketamine/xylazine deep anaesthesia and sacrificed, and their brains were fixed by intracardiac perfusion with $0.9 \%$ saline followed by $4 \%$ paraformaldehyde (PFA). The brain was carefully excised and kept in 4\% PFA for 1 week. After tissue processing, the selected samples were embedded in paraffin. Sections $(4 \mu \mathrm{m})$ were prepared on microscopic slides and then slices were stained with $0.1 \%$ cresyl violet (Lamb; London, UK) that was dissolved in distilled water.

\section{Estimations of covariance and pair correlation function}

For estimation of the local 3-dimensional (3D) arrangements of $\mathrm{PCs}$, transparent lattice bearing a row of points (a set of linear dipole probes with 21-26 intervals each row consisted of 50 points and 49 equidistant intervals) was used. A distance of point interval was $2.6 \mu \mathrm{m}$ on the scale of the tissue. Consequently, both end points of dipoles (DPs) of class size $r=1$ (equivalent to $2.6 \mu \mathrm{m}$ ) had chances of being included within the same cell section. The lattice was superimposed on the live image on a monitor connected to a microscope (Nikon, E-200, Japan). It was set to be random in position and orientation in each field. For each cerebellum, 10 trials with a total of 500 test points were conducted. For every trial, the nature of the tissue component underlying each test point was noted and all this information was recorded on a $50 \times 10$ matrix printed as a blank table with 50 columns and 10 rows. Within the cells of this matrix, each point was coded as 1 (PCs) and 2 (neuropil) (Fig. 2). The covariance of a component (X) was estimated by the following equation [18]: $C(r) X=$ $\Sigma D P(X, r) / \Sigma D P($ ref, r), where $D P(X, r)$ and DP (ref, r) are the dipole length which hit the favoured structure and the nervous tissue. Pair correlation function is the normalised covariance function obtained by dividing covariance by the reference value (squared volume fraction) [18]: $g(r)=C(r) / v v 2$.

\section{Estimations of cross correlation function}

The term cross-correlation function is used to express how different particles are clustered or dispersed together. The cross correlation function was estimated by the following equation [18]: $\mathbf{g}(\mathbf{r}) \mathbf{X Y}=$ $C(r) X Y / V v(X$, ref $) \times V v(Y$, ref $)$. 


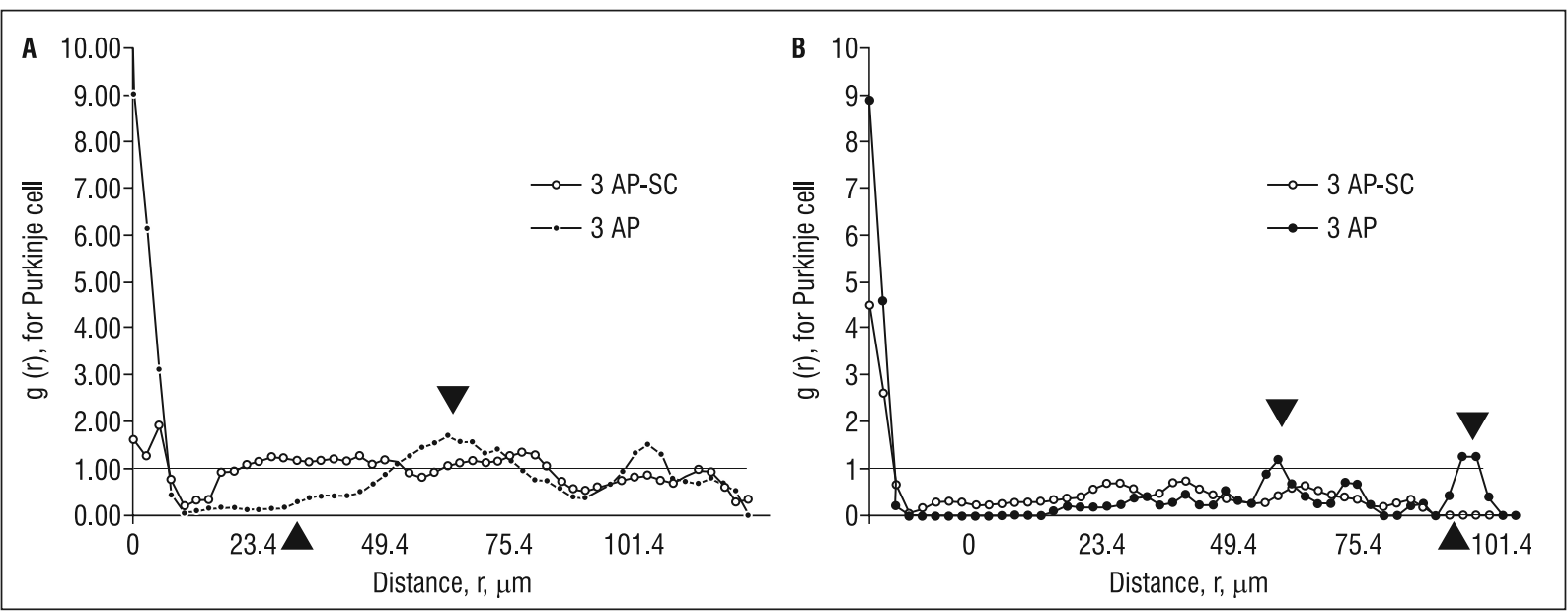

Figure 3. Pair correlation (A), and cross correlation (B) functions $g(r)$ of the Purkinje cells (PCs). In each plot the mean over four cases is plotted with a point-wise confidence interval based on Student's t-distribution. The horizontal reference line corresponded to the values expected for a random spatial arrangement $[g(r)=1]$. The pair correlation function approached closely to the reference line because no significant correlation was found between the values of points lying far away in the cerebellar tissue. The relationship between pair correlation function and dipole distance for PCs in the 3-acetylpyridine (3-AP) and 3-AP-Sertoli cells (SCs) groups was shown. The cross-correlation, $g(r)$, between the PCs plotted versus distance, $r$, in micrometres in the 3-AP and 3-AP-SC groups. The dots are the mean $\mathrm{g}(\mathrm{r})$ of the 6 animals in the 3-AP and 3-AP-SC groups. The horizontal reference line at $\mathrm{g}(\mathrm{r})=1.0$ is the reference line expected for a 'random' structure. " $\boldsymbol{\nabla}$ " and " $\boldsymbol{\Delta}$ " indicate $\mathrm{g}_{\max }$ and $\mathrm{g}_{\text {min }^{\prime}}$ respectively in both groups.

\section{Immunohistochemistry}

Immunohistochemistry (IHC) staining of the cerebellar sections was performed to demonstrate the neuroprotective effect of SCs transplantation in the cerebellum. As explained in tissue preparation, microscopic slides were prepared for IHC. Slides were treated with $3 \% \mathrm{H}_{2} \mathrm{O}_{2}$ for peroxidase inactivation, heated in $10 \mathrm{mM}$ citrate buffer (with $0.05 \%$ Tween20) for antigen retrieval and blocked with $1 \%$ blocking solution ( $1 \%$ BSA and $0.1 \%$ Triton X-100 in PBS). Then, the sections were incubated overnight at $4^{\circ} \mathrm{C}$ with the anti-calbindin (Abcam, 1:200). The following day, sections were incubated with Alexa Fluor 488-conjugated anti-mouse IgG (1:1000) for $2 \mathrm{~h}$ at room temperature. DAPI (4' 6 '-diamidino-2-phenylindole) (molecular probes) was applied to stain nuclei. The sections were washed with $0.1 \mathrm{M}$ PBS, pH 7.4, for 5 min following per step. The slices were analysed using a confocal laser-scanning microscope (LSM 5 PASCAL). As a negative control, the same procedure was done using a similar protocol except for the omission of incubation with the primary antibody. All negative control sections revealed negative reactions.

\section{Data analysis}

Data were revealed as means \pm standard deviation and group mean and coefficients of variation be- tween rats were computed for PCs in the cerebellum. Comparisons of $g(r)$ of the PCs between the groups were done using of Mann-Whitney U-test.

\section{RESULTS}

\section{Spatial arrangement of Purkinje cells}

The data points in 3-AP and 3-Ap-SC groups showed a random arrangement at larger distances between PC. Therefore, the spatial arrangement of the $P C s$ was changed and not normally arranged after 3-AP and dissociation of the PC could be seen at some places. According to the results, fewer PCs and wider gaps were detected in the 3-AP group in comparison of the 3-AP-SC group and $g(r)$ of the 3-AP vs. 3-AP/SC shows significant differences $(p<0.05)$ (Fig. 3).

\section{Immunohistochemistry}

We defined the effect of SCs transplantation on the architecture of neuron in the cerebellum and, in particular, the PCs, because the calbindin protein is a cytosolic protein confined in the PCs. Cell bodies of PCs in the 3-AP group were showed to have the lowest cell density. This investigation was approved by quantitative analysis of the number of PCs that expressed calbindin (Fig. 4F). These results demonstrate that differences between the two ataxic groups and the control and sham groups were significant and the difference between 3-AP 
A
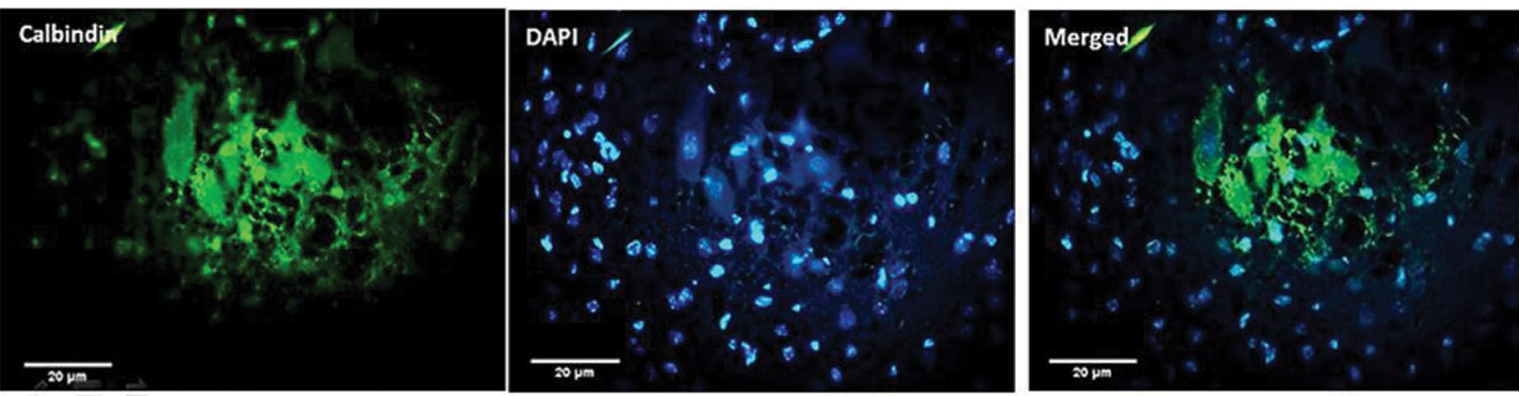

B
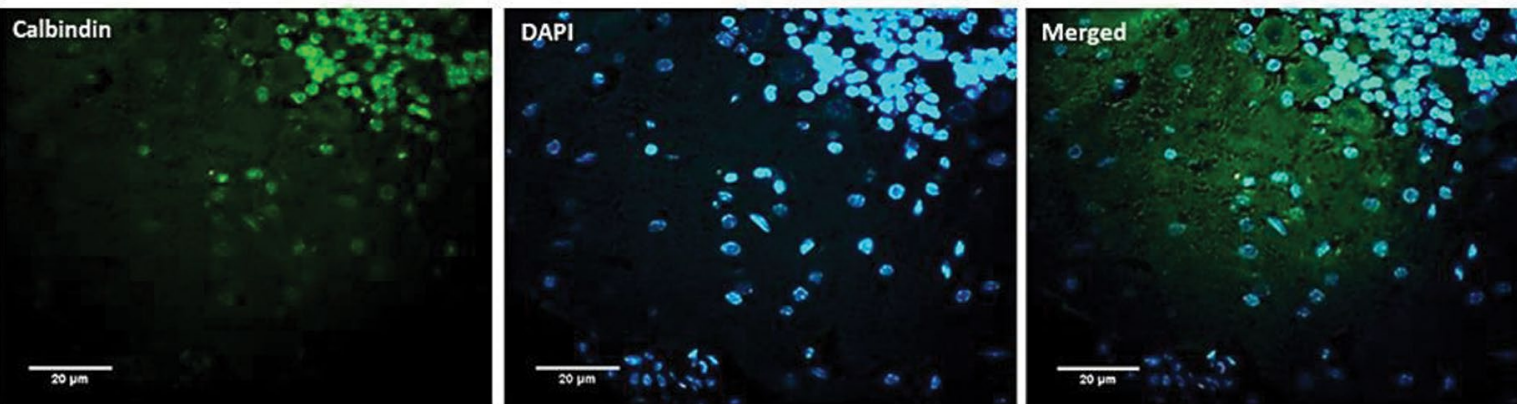

C Calbindin
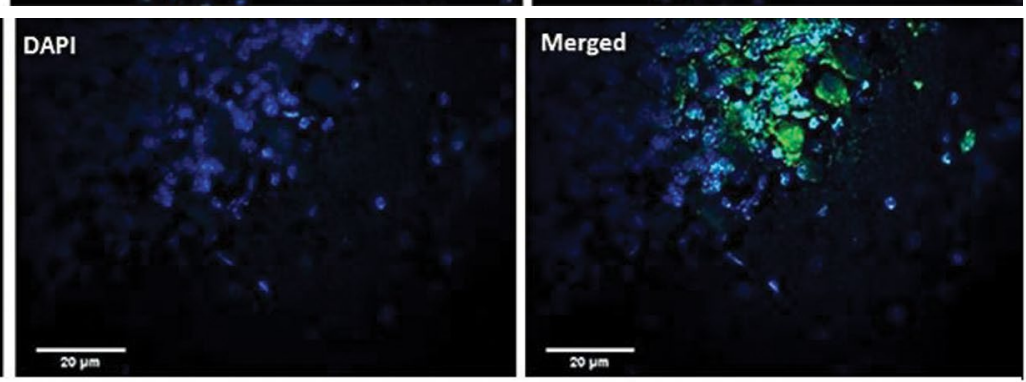

D
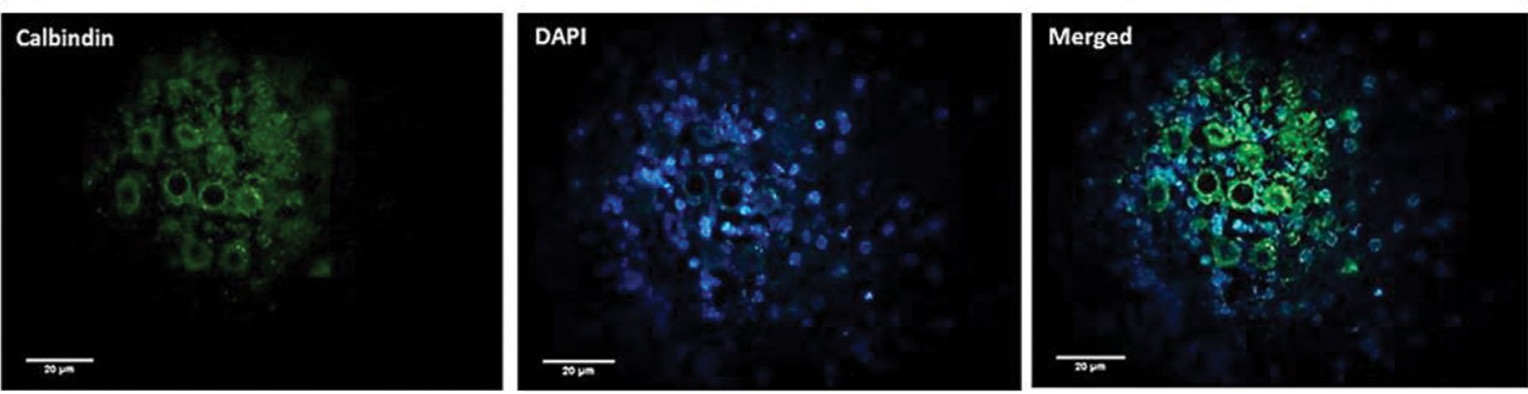

E
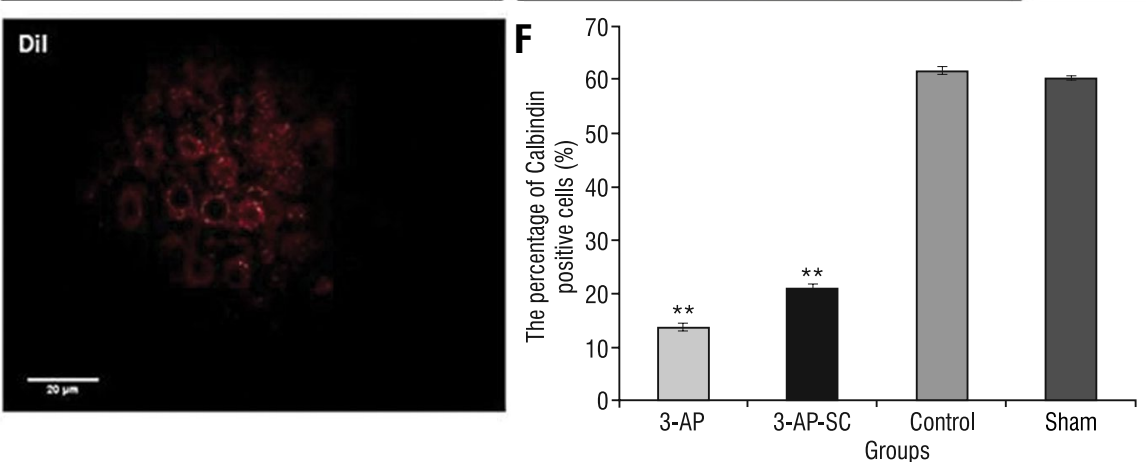

Figure 4. Immunohistochemistry (IHC) on rats' cerebellum sections. Nuclei have been visualised by DAPI (4' 6 '-diamidino-2-phenylindole) staining. A. Control; B. Sham; C. 3-acetylpyridine (3-AP); D. 3-AP-Sertoli cells (SCs) groups. Representative image of Dil (1,1'-dioctadecyl$-3,3,3^{\prime}, 3^{\prime}$-tetramethyl-indocarbocyanine perchlorate) labelled neurons was shown in panel E. F. Quantitative analysis of IHC $(* * p<0.01)$. 
group and 3-AP-SC group is statistically significant $(p<0.01)$.

In the cerebellum, Dil trace was detected and we observed Dil-labelled SCs (Fig. 4).

\section{DISCUSSION}

The current study showed alterations in PCs arrangement using stereological methods. Stereological techniques were used to evaluate the spatial arrangement of PCs and the effect of SCs transplantation on PCs spatial arrangement in ataxic rat's cerebellum. The major advantage of using stereological methods is obtaining precise and accurate estimation and unique quantification of the spatial pattern organisation of the PCs cytoarchitecture. Using modern stereology with 3D probes enables us to determine the distribution of PCs [21].

Previous studies have shown that IP injection of 3-AP cause selective degeneration of ION neurons and subsequently cerebellum loses innervations and inputs from CF and as a result ataxia is induced. The climbing fibres make synapses with PCs and these cells are the exclusive neurons that export data from the cerebellar cortex to cerebellar nuclei and then to the other parts of CNS $[2,7]$. Following the destruction of ION, the axons of these neurons that connect with PCs, also change. Changes such as neuronal structure are impressed. The response of target neurons to the damage include complex changes leading to atrophy or cell death [10]. The synaptic detachment of ION axon terminals to PCs result in a regressive change and a lack of synaptic connection with the efferent destination may lead to trans-neuronal degeneration of the PCs. Purkinje cells are formed only during early CNS development and there is no evidence of their formation after birth [22]. In this study, the potential of using SCs to protect PCs from degeneration in ataxia was explored.

Sertoli cells have been subject of studies on cell therapy of some neurodegenerative diseases $[8,11$, 20]. All these findings suggest that SCs transplantation can be a potential strategy for neurodegenerative disease treatment.

In the present study, the possible neuroprotective effect of SCs against 3-AP-induced ataxia was examined. Decreasing CF has a negative effect on the survival of PC and plasticity of the cerebellar cortex and ataxic rats show cerebellar abnormality including lower cellular density of cortical neurons $[2,9]$. According to our results, loss of the PCs and wider gaps between PCs were detected in the 3-AP rats. Assessments of the PCs in the 3-AP and 3-AP-SC groups indicated more distribution and repulsion in the 3-AP groups. Plots of pair-correlation function showed a wider gap in the spatial arrangement of PCs in the cerebellum after 3-AP administration. The wider gap between PCs should be filled with neuropil. Our results support the hypothesis that an altered pattern of PCs position is present in the cerebellum after 3-AP administration. Our previous study revealed a difference in the neurons and glial cells spatial patterns in medial prefrontal cortex after chronic stress. Interesting data on structural changes that cannot be detected only by qualitative analysis can be obtained by evaluation of the spatial arrangement [18].

Previous studies demonstrate that SCs secrete several neurotropic factors that can provide neurotrophic protection [12] and these factors are involved in decreasing apoptosis and suppress local inflammation and immune activity [23]. Our findings confirm the PCs regenerative potential and unique plasticity in response to SCs transplantation and show that SCS has the capability to protect neurons.

The present study was the first study on neuroprotective effect of SCs transplantation on spatial arrangement of $P C$ disability of cerebellar ataxia induced by 3-AP in rat. The results showed that 3-AP induced cerebellar ataxia in rats and caused degenerative changes in the cerebellum and ION. There is little information about the mechanism of SCs function in the nervous tissue, but its effect on the CNS may involve secretion of trophic factors following SCs transplantation that can improve PC survival after transplantation. Further studies need to be done to investigate the mechanism of SCs preventive and regenerative effects on ataxic nerve system.

\section{CONCLUSIONS}

The following can be concluded on the basis of results obtained from the immunohistochemical and stereological analysis in this study: (1) 3-AP toxin application leads to morphological and neuronal changes in the cerebellum; (2) SCs transplantation in ataxic rats causes partial recovery in the PCs spatial arrangement. In conclusion, SCs transplantation can improve spatial arrangement of PCs in cerebellar ataxia induced by 3-AP in rats.

\section{Acknowledgements}

This work was performed at the Department of Biology and Anatomical Sciences, Shahid Beheshti University 
of Medical Sciences, Tehran, Iran. This study is a part of the thesis written by Rabieh Mohammadi, PhD student of Anatomy. The work was financially supported by the Research Vice-chancellor of Shahid Beheshti University of Medical Sciences (Grant No. 91-6124).

\section{REFERENCES}

1. Akosman MS, Gocmen-Mas N, Karabekir HS. Estimation of Purkinje cell quantification and volumetry in the cerebellum using a stereological technique. Folia Morphol. 2011; 70(4): 240-244, indexed in Pubmed: 22117240.

2. Balaban C. Central neurotoxic effects of intraperitoneally administered 3-acetylpyridine, harmaline and niacinamide in Sprague-Dawley and Long-Evans rats: A critical review of central 3-acetylpyridine neurotoxicity. Brain Res Rev. 1985; 9(1): 21-42, doi: 10.1016/0165-0173(85)90017-7, indexed in Pubmed: 3158380.

3. Barker CF, Billingham RE. Immunologically privileged sites. Adv Immunol. 1977; 25: 1-54, doi: 10.1016/s00652776(08)60930-x, indexed in Pubmed: 345773.

4. Cendelin J. Transplantation and Stem Cell Therapy for Cerebellar Degenerations. Cerebellum. 2016; 15(1): 48-50, doi: 10.1007/s12311-015-0697-1, indexed in Pubmed: 26155762.

5. Chintawar S, Hourez R, Ravella A, et al. Grafting neural precursor cells promotes functional recovery in an SCA1 mouse model. J Neurosci. 2009; 29(42): 13126-13135, doi: 10.1523/JNEUROSCI.0647-09.2009, indexed in Pubmed: 19846700.

6. Dietrichs E. Clinical manifestation of focal cerebellar disease as related to the organization of neural pathways. Acta Neurol Scand Suppl. 2008; 188: 6-11, doi: 10.1111/j.1600-0404.2008.01025.x, indexed in Pubmed: 18439215.

7. Gasbarri A, Pompili A, Pacitti C, et al. Comparative effects of lesions to the ponto-cerebellar and olivo-cerebellar pathways on motor and spatial learning in the rat. Neuroscience. 2003; 116(4): 1131-1140, doi: 10.1016/s03064522(02)00780-7, indexed in Pubmed: 12617954.

8. Hemendinger R, Wang J, Malik S, et al. Sertoli cells improve survival of motor neurons in SOD1 transgenic mice, a model of amyotrophic lateral sclerosis. Exp Neurol. 2005; 196(2): 235-243, doi: 10.1016/j.expneurol.2005.07.025, indexed in Pubmed: 16242126.

9. Jiang $Y Y$, Cao BB, Wang $X Q$, et al. Cerebellar ataxia induced by $3-A P$ affects immunological function. Neuro Endocrinol Lett. 2015; 36(3): 246-256, indexed in Pubmed: 26313392

10. Kemp KC, Cook AJ, Redondo J, et al. Purkinje cell injury, structural plasticity and fusion in patients with Friedreich's ataxia. Acta Neuropathol Commun. 2016; 4(1): 53, doi: 10.1186/s40478-016-0326-3, indexed in Pubmed: 27215193.

11. Liu HW, Kuang YJ, Wu JC, et al. Intrastriatal transplantation of Sertoli cells may improve amphetamine-induced rotation and tyrosine hydroxylase immunoreactivity of the striatum in hemiparkinsonian rats. Brain Res. 1999; 838(12): 227-233, indexed in Pubmed: 10446339.

12. Ma W, Wang C, Su Y, et al. Expression of nerve growth factor and its receptor, tyrosine kinase receptor $A$, in rooster testes. Anim Reprod Sci. 2015; 161: 40-46, doi: 10.1016/j. anireprosci.2015.08.001, indexed in Pubmed: 26297622.

13. Maddocks S, Setchell BP. Recent evidence for immune privilege in the testis. J Reprod Immunol. 1990; 18(1): 9-18, doi: 10.1016/0165-0378(90)90021-w., indexed in Pubmed: 2213734.

14. Manto $M$, Haines $D$, Manto $M$. The cerebellum, cerebellar disorders, and cerebellar research--two centuries of discoveries. Cerebellum. 2008; 7(4): 505-516, doi: 10.1007/ s12311-008-0063-7, indexed in Pubmed: 18855093.

15. Marmolino D, Manto M. Past, present and future therapeutics for cerebellar ataxias. Curr Neuropharmacol. 2010; 8(1): 41-61, doi: 10.2174/157015910790909476, indexed in Pubmed: 20808545.

16. Matsuura S, Shuvaev AN, lizuka A, et al. Mesenchymal stem cells ameliorate cerebellar pathology in a mouse model of spinocerebellar ataxia type 1. Cerebellum. 2014; 13(3): 323-330, doi: 10.1007/s12311-013-0536-1, indexed in Pubmed: 24242763.

17. Mattfeldt T, Eckel S, Fleischer F, et al. Statistical analysis of reduced pair correlation functions of capillaries in the prostate gland. J Microsc. 2006; 223(Pt 2): 107-119, doi: 10.1111/j.1365-2818.2006.01604.x, indexed in Pubmed: 16911071.

18. Noorafshan A, Abdollahifar MA, Karbalay-Doust S. Stress changes the spatial arrangement of neurons and glial cells of medial prefrontal cortex and sertraline and curcumin prevent it. Psychiatry Investig. 2015; 12(1): 73-80, doi: 10.4306/ pi.2015.12.1.73, indexed in Pubmed: 25670949.

19. Paxinos G, Watson C. The Rat Brain in Stereotaxic Coordinates: Hard Cover Edition. Academic Press. 2006: 451.

20. Rodriguez Al, Willing AE, Saporta S, et al. Effects of Sertoli cell transplants in a 3-nitropropionic acid model of early Huntington's disease: a preliminary study. Neurotox Res. 2003; 5(6): 443-450, doi: 10.1007/bf03033174, indexed in Pubmed: 14715448.

21. Schmitz C, Grolms N, Hof PR, et al. Altered Spatial Arrangement of Layer $V$ Pyramidal Cells in the Mouse Brain following Prenatal Low-dose X-Irradiation. A Stereological Study using a Novel Three-dimensional Analysis Method to Estimate the Nearest Neighbor Distance Distributions of Cells in Thick Sections. Cerebral Cortex. 2002; 12(9): 954-960, doi: 10.1093/cercor/12.9.954.

22. Weimann JM, Charlton CA, Brazelton TR, et al. Contribution of transplanted bone marrow cells to Purkinje neurons in human adult brains. Proc Natl Acad Sci U S A. 2003; 100(4): 2088-2093, doi: 10.1073/pnas.0337659100, indexed in Pubmed: 12576546.

23. Willing AE, Cameron DF, Sanberg PR. Sertoli cell transplants: their use in the treatment of neurodegenerative disease. Mol Med Today. 1998; 4(11): 471-477, doi: 10.1016/s13574310(98)01355-0, indexed in Pubmed: 9857366. 